\title{
Brightness function: Binocular versus monocular stimulation'
}

JOSEPH C. STEVENS

JOHN B. PIERCE FOUNDATION LABORATORY AND YALE UNIVERSITY

A dozen observers matched numbers to the apparent brightness of a target viewed by one eye or by both eyes. Brightness grew as a power function of luminance, and the functions were practically identical for the two modes of viewing. Throughout its course, the obtained binocular function tended to fall about a decibel above the monocular function. This small degree of binocular summation, of the order of a jnd, may or may not be significant.

The aim was to learn whether lights look brighter when viewed with two eyes than when viewed with only one. A further aim was to study binocular summation over a wide range of luminances. In the domain of hearing it has long been recognized that a given stimulus level sounds louder when heard by two ears than when heard by only one (Fletcher \& Munson, 1933). Summation is relatively small (equivalent to about $3 \mathrm{~dB}$ ) near the absolute threshold, but at higher levels it may exceed $10 \mathrm{~dB}$ and produce double the apparent loudness (Reynolds \& S. S. Stevens, 1960).

One of the several methods used by Reynolds and Stevens to measure binaural summation was to obtain magnitude estimates of the loudness of various levels of white noise presented sometimes to one ear and sometimes to both. The two sets of estimations generated clearly different psychophysical functions for one- and two-ear listening. Both functions could be described by the general psychophysical power function that is obeyed by so many sensory continua (S. S. Stevens, 1961):

$$
\psi=k(\emptyset-\not)^{\beta}
$$

where $\psi$ is subjective magnitude, $\emptyset$ is physical magnitude, and $\emptyset_{0}$ is the absolute threshoid. For binaural listening the constants $k$ and $\emptyset_{0}$ were smaller and $\beta$ was larger than for monaural listening.

Brightness also obeys Eq. (1) (J. C. Stevens \& S. S. Stevens, 1963), and if binocular summation takes place, a change is to be expected in one or more of the same constants. In an unpublished study, Hammer, Slawson, and Kieffer (1961) found by the method of magnitude estimation that the constants of the brightness function are nearly the same whether the target light is viewed monocularly or binocularly. The present study, which was a refinement of their study, also demonstrates the near invariance of the constants of the power function. In other words, brightness fails to exhibit the substantial summation found in the sense of hearing.

\section{Procedure}

The experiment was conducted in a dark booth. From a distance of $66 \mathrm{~cm}$ the observer (O) viewed a $3^{\circ}$ circular target produced by a diffusing screen illuminated by a $150 \mathrm{~W}$ projector lamp located outside of the booth. The luminance of the target was varied by means of neutral density filters. The levels presented ranged from 44 to $104 \mathrm{~dB}$ re $10^{-10} \mathrm{~L}(2.5$ to $\left.2.5 \times 10^{6} \mu \mathrm{L}\right)$.

The $O$ viewed the target through an eyepiece that held a shutter in front of each eye. These shutters were operated by remote control from outside of the booth. Before a presentation of the target, either or both of the shutters were opened. Then the projector lamp was lighted for $1 \mathrm{sec}$. Each of seven luminance levels was presented four times in the course of the experimental session, once to the left eye, once to the right eye, and twice to both eyes. The order of presentation of the stimulus levels and the mode of viewing (monocular or binocular) were irregular and different from one $O$ to another. The time between presentations was about $30 \mathrm{sec}$.

The first stimulus was presented binocularly and had a luminance of either $64 \mathrm{~dB}$ (four Os), $74 \mathrm{~dB}$ (four Os), or $84 \mathrm{~dB}$ (four $\mathrm{Os}$ ). The $\mathrm{O}$ was instructed to assign to the first stimulus whatever number seemed to him appropriate to represent the apparent brightness, and to assign numbers to subsequent stimuli in proportion to their apparent brightness (the method of magnitude estimation). The first stimulus was presented twice more (binocularly) later in the session; the very first assignment was not counted.

Not until after the session ended was the $O$ informed that the target had been viewed sometimes monocularly and other times binocularly. Of the 12 Os who took part, seven said they had been unaware that the mode of viewing varied, three said they inferred that the mode changed from time to time (presumably from the sound of the shutters) but were unable to tell after the target presentation what the mode had been, and two said that they occasionally experienced a momentary double image at the onset of a binocular presentation.

\section{Resulis}

Table 1 shows the averages (log) and the variability of the estimates for each stimulus level under each mode of viewing (binocular, pooled data for left and right eyes, and left and right eyes separately). The first row gives the mean $(\bar{x})$ of the logarithmically 
Table 1. The mean $(\bar{x})$, variance $\left(\sigma^{2}\right)$, and variance corrected by removing the portion attributable to modulus differences $\left(\sigma_{c}{ }^{2}\right)$, for the logaritims of the estimates of each stimulus.

\begin{tabular}{|c|c|c|c|c|c|}
\hline $\begin{array}{l}\text { dB re } \\
10-10\end{array}$ & & Binocular & $\begin{array}{l}\text { Monocular } \\
\text { (left \& right) }\end{array}$ & Left eye & Right eye \\
\hline \multirow{3}{*}{44.0} & \multirow{3}{*}{$\begin{array}{l}\bar{x} \\
\sigma^{2} \\
\sigma_{c}{ }^{2}\end{array}$} & 0.0584 & -0.1032 & -0.0540 & -0.1524 \\
\hline & & 0.0280 & 0.1459 & 0.1519 & 0.1350 \\
\hline & & 0.0703 & 0.0843 & 0.0937 & 0.0691 \\
\hline \multirow{3}{*}{54.0} & \multirow{3}{*}{$\begin{array}{l}\bar{x}_{2} \\
\sigma^{2} \\
\sigma_{c}{ }^{2}\end{array}$} & 0.4350 & 0.4083 & 0.4195 & 0.3972 \\
\hline & & 0.1127 & 0.1321 & 0.1764 & 0.0874 \\
\hline & & 0.0511 & 0.0651 & 0.0720 & 0.0355 \\
\hline \multirow{3}{*}{64.0} & \multirow{3}{*}{$\begin{array}{l}\bar{x}_{2} \\
\sigma^{2} \\
\sigma_{c}^{2}\end{array}$} & 0.8320 & 0.7545 & 0.7172 & 0.7918 \\
\hline & & 0.0483 & 0.0616 & 0.0491 & 0.0713 \\
\hline & & 0.0294 & 0.0280 & 0.0459 & 0.0142 \\
\hline \multirow{3}{*}{74.0} & \multirow{3}{*}{$\begin{array}{l}\bar{x} \\
\sigma^{2} \\
\sigma_{c}{ }^{2}\end{array}$} & 1.0891 & 1.1123 & 1.1510 & 1.0737 \\
\hline & & 0.0517 & 0.1001 & 0.1408 & 0.0564 \\
\hline & & 0.0130 & 0.0357 & 0.0314 & 0.0334 \\
\hline \multirow{3}{*}{84.0} & \multirow{3}{*}{$\begin{array}{l}\bar{x} \\
\sigma^{2} \\
\sigma_{c} 2\end{array}$} & 1.3746 & 1.3188 & 1.2925 & 1.3450 \\
\hline & & 0.0767 & 0.1036 & 0.1154 & 0.0904 \\
\hline & & 0.0185 & 0.0290 & 0.0249 & 0.0270 \\
\hline \multirow{3}{*}{94.0} & \multirow{3}{*}{$\begin{array}{l}\bar{x} \\
\sigma^{2} \\
\sigma_{c}{ }^{2}\end{array}$} & 1.5789 & 1.5678 & 1.5553 & 1.5803 \\
\hline & & 0.0527 & 0.0806 & 0.0828 & 0.0781 \\
\hline & & 0.0123 & 0.0246 & 0.0360 & 0.0126 \\
\hline \multirow{3}{*}{104.0} & \multirow{3}{*}{$\begin{array}{l}\bar{x} \\
\sigma^{2} \\
\sigma_{c}{ }^{2}\end{array}$} & 1.8728 & 1.7739 & 1.7540 & 1.7938 \\
\hline & & 0.0910 & 0.0745 & 0.0529 & 0.0954 \\
\hline & & 0.0213 & 0.0169 & 0.0164 & 0.0183 \\
\hline
\end{tabular}

transformed estimates (the antilogarithm of $\bar{x}$ is the geometric mean). The second row is the variance $\left(\sigma^{2}\right)$ of the logarithmically transformed estimates. A substantial portion of this variance is attributable to the fact that each $O$ was free to adopt his own modulus, 1.e., to decide what number to call the first stimulus presented. This portion of the variability can be partialled out by a procedure suggested by Lane, Catania, and S. S. Stevens (1961). First, the grand mean of all the logarithmically transformed estimates for a given mode of viewing is computed, then the mean of the estimates for each $O$ separately. For each individual, the difference between the grand mean and individual mean is then calculated and added to all of his estimates. This procedure leaves the values of $\bar{x}$ unchanged but removes the variance attributable to choice of modulus. The remaining variance $\left(\sigma_{c}{ }^{2}\right)$, given in the third row, is about $40 \%$ as large as the total variance $\left(\sigma^{2}\right)$, so that differences in modulus appear to account for almost $60 \%$ of the variance in these magnitude estimates.

The geometric means of the estimates of each stimulus made under each mode of viewing are plotted in the $\log -\log$ coordinates of Fig. 1. Figure 1 contains two abscissas. The upper one is the stimulus in $\mathrm{dB}$ re $10^{-10} \mathrm{~L}$. Plotted against this scale the estimates fit a linear function fairly well, except at the low end, near the absolute threshold, where the brightness function turns down sharply.

The straight line portion of the function in Fig. 1 fits the equation:

$$
\log \psi=\log k+0.27 \log \mathbf{B}
$$

where $\psi$ is brightness and $B$ is luminance. In its nonlogarithmic form Eq. (2) becomes:

$$
\psi=\mathbf{k B}^{0.27} \text {. }
$$

The slope (or exponent) 0.27 is a little smaller than the value 0.33 , believed, on the basis of a large number of studies, to govern the brightness function (J. C. Stevens \& S. S. Stevens, 1963). The method of magnitude estimation may sometimes yield a rather low value of the exponent (S. S. Stevens \& Greenbaum, 1966).

When a value approximating the absolute threshold, $B_{O}$, is subtracted from each luminance value, the equation of the power function becomes

$$
\log \psi=\log k+0.27 \log \left(B-B_{0}\right)
$$

or in its nonlogarithmic form,

$$
\psi=k\left(B-B_{O}\right)^{0.27} \text {. }
$$

The subtraction of $B_{o}$ converts the stimulus scale into a scale of "distance" above the absolute threshold, for the zero of the stimulus scale is set to coincide

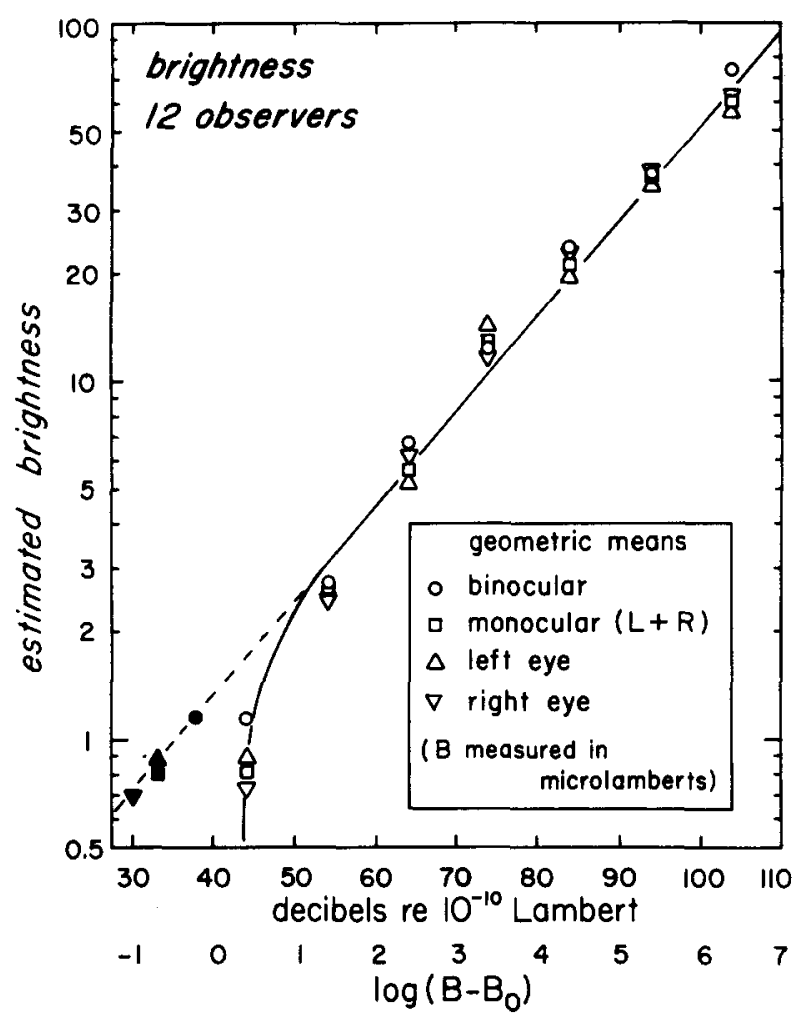

Fig. 1. Apparent brightness: monocular vs binocular estimations. Untilled symbols: estimates plotted against upper abscissa. Filled symbols: estimates plotted against lower abscissa. The subtraction of the threshold constant $B_{0}$ (lower abscissa) has a noticeable effect on only the lowest set of points (see text). 
Table 2. The threshold correction $B_{0}$, the exponent $\beta$, the constant $k$, and the Pearson coefficient of correlation $r$ for the best-fitting power function of the form $\psi=\mathbf{k}\left(\mathbf{B}-\mathbf{B}_{\mathbf{0}}\right) \beta$ when $\mathbf{B}$ is measured in microlamberts. (Only the value of $k$ is influenced by the choice of unit for the measurement of $B$.)

\begin{tabular}{|c|c|c|c|c|}
\hline & $B_{0}$ & $\beta$ & $\mathbf{k}$ & $r$ \\
\hline $\begin{array}{l}\text { Binocular } \\
\text { Monocular } \\
\text { (left \& right) }\end{array}$ & $\begin{array}{l}1.9 \\
2.3\end{array}$ & $\begin{array}{l}0.276 \\
0.272\end{array}$ & $\begin{array}{l}1.32 \\
1.25\end{array}$ & $\begin{array}{l}0.998 \\
0.997\end{array}$ \\
\hline $\begin{array}{l}\text { Left eye } \\
\text { Right eye }\end{array}$ & $\begin{array}{l}2.3 \\
2.4\end{array}$ & $\begin{array}{l}0.263 \\
0.271\end{array}$ & $\begin{array}{l}1.33 \\
1.29\end{array}$ & $\begin{array}{l}0.995 \\
0.998\end{array}$ \\
\hline
\end{tabular}

with the threshold (J.C. Stevens \& S.S. Stevens, 1962). In the present experiment, the value of $B_{O}$ is large relative to the lowest stimulus $(44 \mathrm{~dB})$ but practically negligible compared to the next higher stimulus $(55 \mathrm{~dB})$. Consequently, when the magnitude estimates are plotted as a function of $\mathrm{B}-\mathrm{B}_{\mathrm{O}}$ (lower abscissa), only the lowest points of Fig. 1 (filled symbols) are noticeably affected. Use of the lower abscissa removes most of the curvature in Fig. 1.

Investigations of the form of the psychophysical function in the vicinity of the threshold have shown that Eq. (1) is able to describe the growth of apparent magnitude on a variety of sensory continua.

Four equations having the form of $\mathrm{Eq}$. (1) were fitted separately to the data for the left eye, for the right eye, for the left and right eyes combined, and for binocular viewing. The obtained values of $B_{0}, \beta$, and $k$ are listed in Table 2, from which it is apparent that the constants of the brightness function are practically the same for all four sets of data.
Since a least squares solution does not exist for Eq. (1), the values of the constants had to be obtained by an iterative process. This process involves the following steps. First, a large number of possible values of $B_{o}$ are assumed. For each value assumed, a least squares solution is obtained for $k$ and $\beta$, operating on $\bar{x}$ and $\log \left(B-B_{0}\right)$. Second, for each least squares solution, a Pearson correlation coefficient $r$ is obtained to show the degree of relation between $\bar{x}$ and $\log \left(B-B_{0}\right)$. Third, the values of $r$ obtained are plotted as a function of $\mathrm{B}_{\mathrm{O}}$. One such plot (for the binocular data) is shown in Fig. 2, indicating for those data that the maximum correlation $(0.998)$ is obtained when $B_{O}$ has a value of $1.9 \mu \mathrm{L}\left(42.9 \mathrm{~dB}\right.$ re $\left.10^{-10} \mathrm{~L}\right)$. Figure 2 also shows that when $B_{o}$ equals $1.9, \beta$ equals 0.276 . In other words, the power function

$$
\psi=1.32(\mathrm{~B}-1.9)^{0.276}
$$

is the one that fits the binocular data best. All the values in Table 2 were obtained by the iterative process.

With the constants from Table 2, two brightness functions, one for the binocular data, the other for the pooled monocular data, were constructed in Fig. 3. These show a tiny difference, of the order of $1 \mathrm{~dB}$, in favor of the binocular function. It is debatable whether a difference of this order of magnitude should be taken as serious evidence for binocular summation. A difference of $1 \mathrm{~dB}$ is roughly the size of a jnd measured under comparable viewing conditions. Moreover, the difference could have resulted from a small potential disparity between the states of adaptation under monocular and binocular viewing. For example,

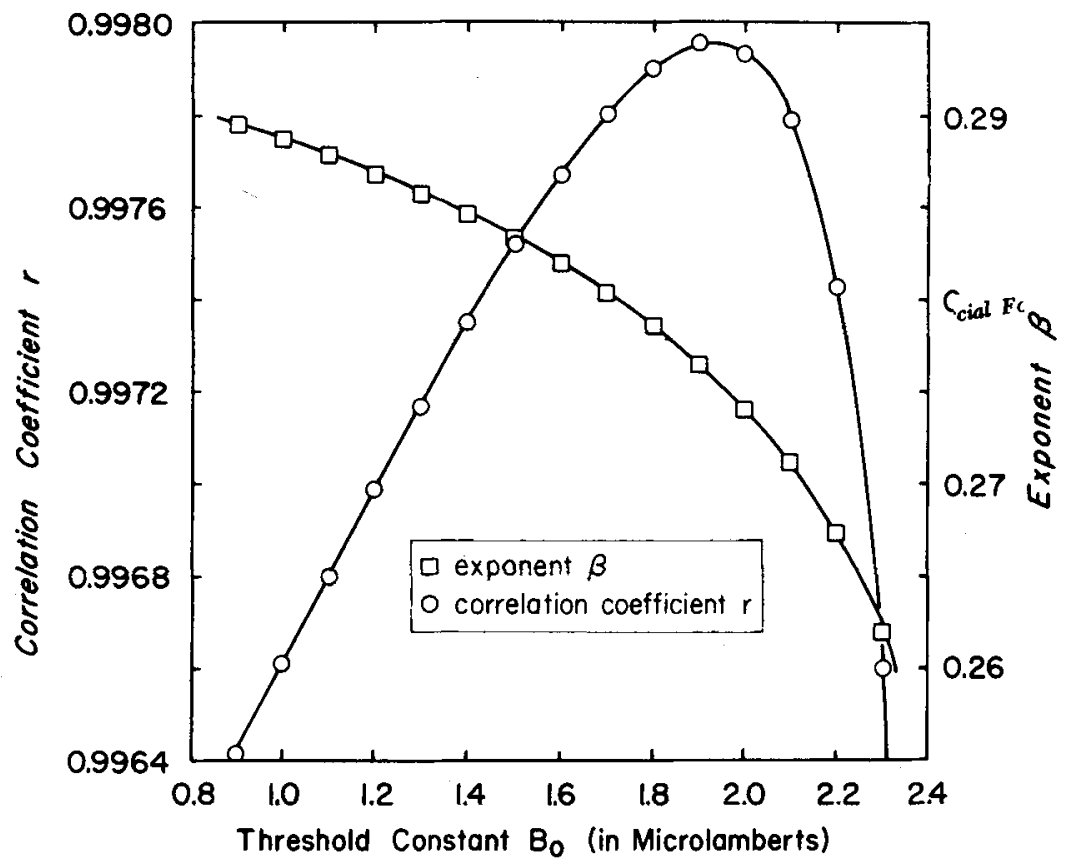

Fig. 2. How the correlation coefficient $r$ and the exponent $\beta$ depend on the assumed value of the threshold constant $B_{0}$ for the binocular data. 


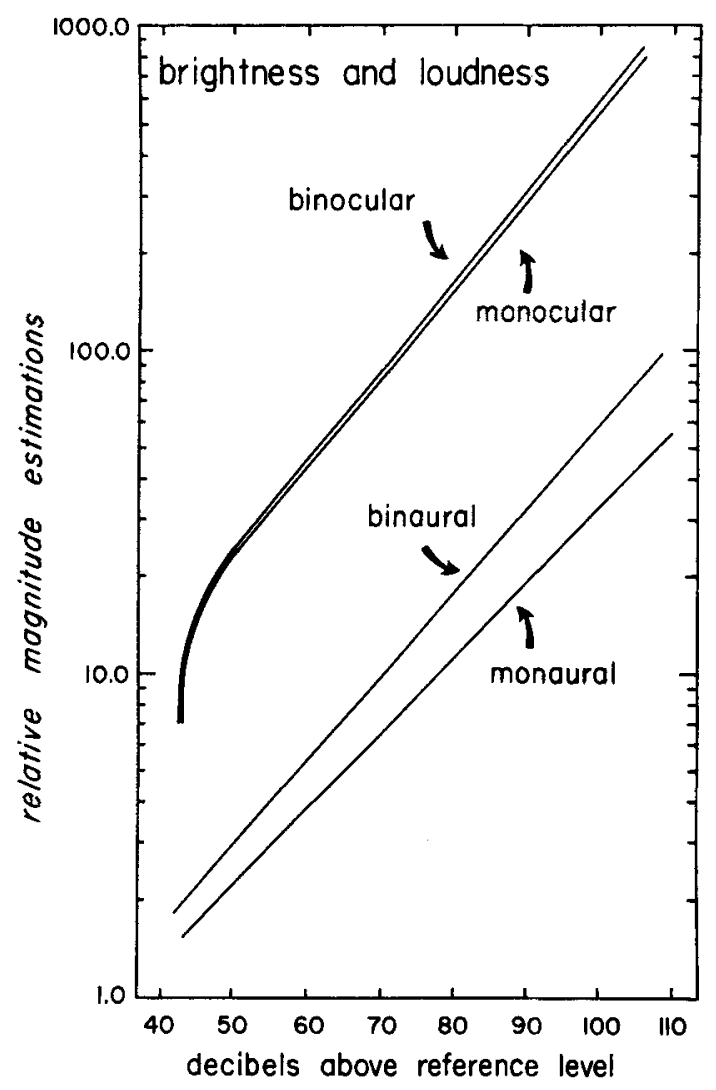

Fig. 3. Loudness and brightness functions determined by the method of magnitude estimation. The loudness functions are redrawn from Reynolds and Stevens (1960). The reference level for hearing: 0.0002 microbar. The reference level for vision: $10^{-10} \mathrm{~L}$.

whenever a monocular presentation followed a binocular presentation, the eye stimulated had always been stimulated in the previous presentation; but whenever a binocular trial followed a monocular trial, one of the eyes had not been stimulated in the previous presentation and therefore had extra time to dark adapt. An analysis of all the sequential probabilities in this experiment shows that the conditions of adaptation would favor binocular viewing to a small degree. There is, however, no way to be sure whether differential adaptation actually played a significant role.

The outcome of this experiment does not, therefore, settle the long standing question whether some degree of binocular summation ever takes place. The method of magnitude estimation may lack the precision necessary to detect a small degree of summation either at a particular luminance level or throughout the dynamic range of brightness. The often conflicting evidence concerning the nature and amount of summation at the threshold has been reviewed and appraised by Matin (1962). Although the threshold was not here explicitly measured by the conventional psychophysical procedures, the present experiment tends to support the usual (but not universal) finding that the binocular threshold is a little lower than the monocular.

Only a few experimental investigations of summation have been aimed at levels above threshold. Levelt (1965) reviewed the literature and contributed a series of interesting experiments on several types of binocular interactions. It would seem that a small degree of summation cannot be ruled out, but the evidence is inconclusive.

In any case, it is clear that binocular summation is minute compared to the large summation that takes place over the whole dynamic range of loudness. The contrast is brought out clearly in Fig. 3, where the monaural and binaural functions determined by the method of magnitude estimation (Reynolds \& S. S. Stevens, 1960) are shown with the brightness functions.

The near invariance of the brightness function has a fortunate practical implication, for it is often convenient to assess the effect on brightness of such parameters as adaptation or glare by matching a criterion target seen by one eye to a test target seen by the other eye. It is helpful to know that the brightness of the criterion target does not depend substantially on whether it is viewed monocularly or binocularly. On the other hand, similar procedures for measuring the effect of one or another parameter on loudness must reckon with the difference between the monaural and binaural loudness functions.

\section{References}

Fletcher, H., \& Munson, W. A. Loudness: Its definition, measure ment, and calculation. J. Acoust. Soc, Amer., 1933, 5, 82-108.

Hammer, L. R., Kieffer, J. D., \& Slawson, A. W. A test of binocular summation. In Laboratory of Psychophysics, Harvard University, Perlod. Status Rep. No. 38, 1961.

Lane, H. L., Catania, A. C., \& Stevens, S. S. Voice level: Autophonic scale, perceived loudness, and effect of sidetone. $J$. Acoust. Soc. Amer., 1961, 33, 160-167.

Levelt, W. J. M. On binocular rivalry. Assen, The Netherlands: Royal VanGorcum Ltd., 1965.

Matin, L. Binocular summation at the absolute threshold of peripheral vision. J. Opt. Soc. Amer., 1962, 52, 1276-1286.

Reynolds, G. S., \& Stevens, S. S. Binaural summation of loudness. J. Acoust. Soc. Amer., 1960, 32, 1337-1344.

Stevens, J. C., \& Stevens, S. S. Physiological zero and the psychophysical law. In Proceedings of the Sixteenth International Congress of Psychology. Amsterdam: North Holland, 1962. Pp. $192-193$.

Stevens, J. C., \& Stevens, S. S. Brightness function: Effect of adaptation. J. Opt. Soc. Amer., 1963, 53, 375-385.

Stevens, S. S. To honor Fechner and repeal his law. Science. $1961,133,80-86$.

Stevens, S. S., Greenbaum, H. B. Regression effect in psychophysical judgment. Percept. \& Psychophys., 1966, 1, 439-446.

\section{Note}

1. The research described in this paper was conducted in the Laboratory of Psychophysics, Harvard University, and was supported in part by the National Science Foundation and in part by the National Institutes of Health.

(Accepted for publication June 7, 1967.) 